Aus der Breslauer chirurgischen Klinik (Direktor: Geheimrat Prof. Dr. H. Küttner).

\title{
Zur Kenntnis der Fremdkörper des Duodenums.
}

Von Dr. Eduard Melchior, Assistent der Klinik.

(Mit 5 Abbildungen.)

Die Mehrzahl aller vom Munde a us in den Magen-Darm. kanal gelangenden Fremdkörper — nur solche sollen im folgenden berücksichtigt werden - verlassen denselben wieder spontan per vias naturales. Andere werden während ihrer Passage arretiert und können dann zu schweren Komplikationen führen. Die Erfahrung hat gelehrt, daß es sich hierbei zumeist um typische mit großer Regelmäßigkeit wiederkehrende Lo$\mathrm{k}$ alisationen handelt. Wie Wölfler und Lieblein in ihrer grundlegenden, aus dem Jahre I909 stammenden Monographie angeben, ist als eine derartige Prädilektionsstelle zunächst der Magen anzusehen, und zwar die Gegend vor dem Pylorus; sodann der unterste Dünndarm unmittelbar vor der Bauhinschen Klappe, sowie das Cöcum. „Schließlich kommt noch in Betracht das Rektum, das oft vielen Fremdkörpern zu längerem Aufenthalte dient und für kleinere Fremdkörper der Processus vermiformis. Finden sich Hernien oder Meckelsche Divertikel vor, so pflegen sich nicht selten die Fremdkörper auch in diesen für längere Zeit festzusetzen. Es ist selbstverständlich, da $B$ in allen Fällen, in welchen wir pathologische Verengerungen im Bereich des Magen-Darmkanals vorfinden, auch vor diesen besonders größere Fremdkörper längere Zeit Halt machen werden." -

Das Duodenum wird in diesem Zusammenhange nicht genant. Immerhin finden sich unter der bei Wölfler und Lieblein mitgeteilten Kasuistik einige Beobachtungen, die sich auf im Zwölffingerdarm zurückgehaltene Fremdkörper 
beziehen. Nach Schloffer ergibt sich hieraus, daß das Steckenbleiben langer Fremdkörper im Duodenum ,ein zwar seltenes, aber doch beobachtetes Vorkommnis darstellt". Auch in der übrigen Literatur wird diese Anschauung geteilt. So schreibt Dehmel: ,Verschluckte Fremdkörper bleiben bei ihrer Passage durch den Magen-Darmkanal sehr selten im Darm und besonders selten im Duodenum stecken." In einer von Gaultier im Jahre I 910 erschienenen Monographie der Duodenalerkrankungen heißt es : „Les corps étrangers du duodénum sont plutôt choses exceptionelles" usw.

Diese angebliche Seltenheit des Vorkommens von Fremdkörpern im Duodenum muß a priori überraschen. Stellt doch anatomisch das Duodenum den am besten fixierten Darmabschnitt dar, der außerdem ausgesprochen winklige Krümmungen aufweist, somit eine Konfiguration, die der Passage von Fremdkörpern mit größeren Längsdurchmessern eigentlich recht erhebliche Schwierigkeiten bieten sollte.

Ein in der Küttnerschen Klinik im letzten Jahre zur Beobachtung gelangter Fall von Fremdkörpern im Duodenum übrigens wohl der erste, bei dem diese Diagnose schon vor der Operation gestellt werden konnte gab uns nun Gelegenheit, diese Frage einmal zu revidieren. Es hat sich hierbei tatsächlich ergeben, daß jene Ansicht von der Seltenheit des Vorkommens von Fremdkörpern im Duodenum nicht mehr haltbar ist. Während nämlich Wölfler und LiebIein in ihrer Monographie insgesamt nur über 12 Enterotomien (mit Ausschluß von Herniotomien) wegen verschluckter Fremdkörper berichten konnten, beträgt die Zahl der von uns gesammelten Fälle, in denen derartige Fremdkörper allein des Duodenums Anlaß zu operativen Eingriffen boten, nicht weniger als 23, d. h. annähernd das Doppelte. Wie auffällig sich die Beobachtungen von Fremdkörpern im Duodenum gemehrt haben, geht auch daraus hervor, daß allein aus dem Jahre I 913 nicht weniger als 5 operativ behandelte Fälle dieser Art bisher vorliegen (Iñigo, Rovsing, Löschke, Geiling, unser Fall).

Wir werden demnach zu der Auffassung gedrängt, da $B$ das Duodenum - im strikten Gegensatze zu den oben wiedergegebenen früheren Anschaungen - 
sogar eine ganz ausgesprochene Prädilektionsstelle für die Arretierung verschluckter Fremdkörper darstellt.

Aber auch in klinisch-prognostischer Hinsicht scheinen die Fremdkörper des Duodenums, wie in der folgenden Darstellung gezeigt werden soll, eine gewisse Sonderstellung einzunehmen. Gerade dieser Umstand war es auch, der uns zu einer spezielleren Bearbeitung dieses Themas Veranlassung gab.

Wir schicken der Darstellung im einzelnen zunächst die Daten unseres persönlichen, in der Küttnerschen Klinik beobachteten Falles voraus.

G. H., 24 jährige Krankenpflegerin. Aufgenommen 15. VII. 1913. Die Patientin wird aus der orthopädischen Abteilung überwiesen, die sie vor einem Monat wegen $S \mathrm{chmerzen}$ in der Gegend der Lendenwirbelsäule aufgesucht hatte. Bei der radiologischen Untersuchung der Wirbelsäule fand sich hierbei als unerwarteter Befund eine rechts und vor dem 4. Lendenwirbel befindliche quergestellte $\mathrm{Nadel}$ (s. Fig. 5). Patientin wurde daher der Klinik überwiesen.

Anamnestisch ergab sich nun, daß Patientin vor 5 Jahren aus unbekannter Ursache ungefähr neun Nähnadeln in einem Nadelbrief verschluckt hat. Sie hatte während des ersten Vierteljahrs starke Schmerzen, die dann vergingen, sich aber gelegentlich nach Indigestionen wieder bemerkbar machten. Im Mai IgIo auswärts wegen chronischer Blinddarmentzündung operiert. Vor einem Monat suchte Patientin wegen Rückenschmerzen die orthopädische Abteilung auf (s. oben). Seit 14 Tagen hat sie fast ununterbrochen Schmerzen in der rechten Bauchseite. Patientin kann im Bett nur auf dem Rücken liegen, da bei der geringsten Bewegung nach der Seite Schmerzen auftreten. $O b$ Nadeln abgegangen sind, ist nicht bekannt.

B ef und: Mittelgroße, kräftig gebaute Person in gutem Ernährungszustande. Brustorgane o. B.

A bdomen: Starker Panniculus adiposus, Leib überall weich. Ein Finger breit rechts vom Nabel ist eine auf Druck sehr schmerzhafte Stelle nachweisbar. Patientin gibt an, daß der Schmerz beim Druck sich von hier aus $\mathrm{nach}$ hinten in den Rücken hinein fortsetzt. Sonst nirgends eine Druckempfindlichkeit. Untersuchung per rectum o. B. Das Röntgen bild - Bauchlage, bei leerem bzw. wenig gefülltem Magen angefertigt - zeigt der druckempfindlichen Stelle entsprechend etwas rechts von der Wirbelsäule in $\mathrm{Höhe} \mathrm{des} \mathrm{3.-2.} \mathrm{Lendenwirbels} \mathrm{drei}$ fast senkrecht stehende $\mathrm{Nadeln}$, die nach unten leicht konvergieren. 
Es wird auf diesen Befund hin als Sit $\mathrm{z}$ der $\mathrm{N}$ adeln die $\mathrm{Pars}$ descendens duodeni angesprochen. Die vier Wochen vorher beobachtete quergestellte Nadel - die anscheinend der Pars horizontalis inferior duodeni angehörte - ist jetzt nicht mehr sichtbar. Ein weiteres, nach Wismutbreifüllung des Magens angefertigtes Röntgenbild - s. Fig 4 - zeigt die drei Nadeln weiter nach rechts und abwärts verschoben, eine vierte Nadel ist etwas weiter oberhalb in unmittelbarer Nähe des Pylorus zu erkennen. Die Diagnose erscheint damit gesichert.

Die Operation wurde am 24. VII. I9I3 von Herrn Privatdozent Dr. Frits ch ausgeführt. Medianschnitt zwei Finger breit unterhalb des Processus xiphoideus bis zwei Finger breit unterhalb des Nabels. Eröffnung des Peritoneums in der gleichen Ausdehnung. Es wird nun zunächst - um alle anderen Eventualitäten auszuschließen - systematisch der Magen, Jejunum, Ileum und Colon abgesucht. Hierbei zeigt sich an der Vorderfläche des Cöcums die Darmwand an einzelnen Stellen etwas narbig verändert. Von den Nadeln selbst ist nichts nachzuweisen. Es bleibt also nur das D u od e num übrig. Bereits bei der Betrachtung fällt hier auf, daß in der Pylorusgegend, am Übergang in die Pars superior duodeni, die Darmwand deutlich verdickt ist. Die Serosa zeigt in diesem Bezirke leichte narbige Veränderungen. In der Pars superior duodeni selbst, die durch starkes Anziehen des Magens nach links zugänglich gemacht wird, ist zunächst ebenfalls nichts von den Nadeln zu fühlen. Erst nachdem das Duodenum mitsamt der Flexura hepatica coli von außen und oben her mobilisiert ist (entsprechend dem von $\mathrm{W}$ ia $\mathrm{rt}$ angegebenen Verfahren) füh $1 \mathrm{t}$ man di e oberen Enden $z$ wei er $\mathrm{Nadeln}$ in der Pars descendens duodeni. Die unteren Enden sind mit dem Finger nicht erreichbar. Der Pankreask opf erscheint geschwollen, sehr hart. Abklemmen des Duodenums nach dem Magen hin und Abtamponieren der freien Bauch. höhle. Dann Längsincision in das Duodenum. Eine Nadel stellt sich sofort ein und wird herausgezogen, eine zweite ist mit dem in das Duodenum eingeführten Finger fühlbar, hat aber das Bestreben, nach abwärts auszuweichen. Es gelingt schließlich mit einiger Schwierig. keit, die Nadel zwischen dem in den Darm eingeführten Zeigefinger und dem außen an der Darmwand liegenden Daumen zu fixieren und dann mit der Pinzette herauszuziehen. Querer Verschluß der Darmöffnung in drei Etagen. Bei nunmehriger Kontrolle der Pars superior duodeni fühlt man plötzlich noch $z$ wei $\mathrm{N}$ a deln in ihrergan $z$ en Länge abtastbar nicht weit vom Pylorus. Es gelingt leicht, diese beiden Nadeln durch die Duodenalwand nach vorn durchzustechen und zu entfernen, worauf die sehr kleinen Darmöffnungen doppelt übernäht werden.

Zur Entlastung der Duodenalnaht und auch, um eine ev. sich 
ausbildende Stenose unschädlich zu machen, wird eine Gastrojejunostomia retrocolica post. mit Knopf angelegt. Schmaler Tampon in die Nähe der Nahtstelle. Verschluß der Bauchhöhle in Etagen.

Der Verlauf war völlig ungestört. Der Knopf ging prompt ab. Eine nochmalige Röntgenaufnahme ließ keine Nadeln mehr erkennen. Patientin wurde am I3. VIII. völlig beschwerdefrei entlassen. - Die vier entfernten Nadeln sind Nähnadeln von $4,4 \mathrm{~cm}$ Länge (Größe „4“ des Handels). Eine derselben ist abgebrochen (s. Fig. I).

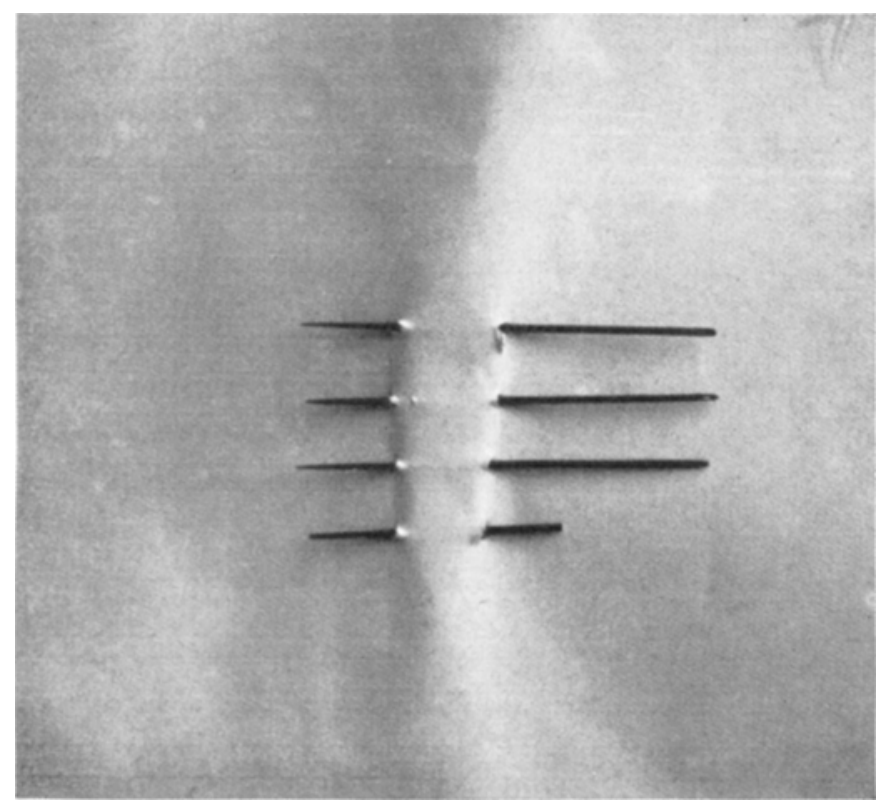

Fig. I.

Außer dieser Beobachtung vermochten wir in der Literatur noch 22 weitere Fälle, in denen Fremdkörper des Duodenum die Ursache zu operativen Eingriffen abgaben, aufzufinden. Dieselben sind am Schlusse dieser Arbeit im Auszuge mitgeteilt. Ein hierunter befindlicher, von Batavyal mitgeteilter Fall (20) war uns allerdings nur dem Titel nach zugänglich. Von Fällen, in denen inkarzerierte Gallensteine die Indikation zur Duodenotomie abgaben - Taylor, Tupper, Pilcher, Wiesinger u.a. - wurde hierbei abgesehen. Das gleiche gilt 
für die Fälle von Haargeschwülsten des Duodenum bzw. sonstiger aus unverdaulichem Material entstehenden Scheintumoren, da unter diesen Umständen die Beteiligung des Magens klinisch im Vordergrunde zu stehen pflegt.

Bezüglich der Art der Fremdkörper beziehen sich die uns hier beschäftigenden Fälle - den obigen theoretischen Vorstellungen entsprechend - fast ausnahmslos auf ausgesprochen längliche Gegenstände:

$\mathrm{N}$ adel $\mathrm{n}$ der verschiedensten Art, wie Nähnadeln, Haar-

nadeln, Hutnadeln usw. (Fall 1, 4, 6, 7, 13, 15, I8, 19, 20, 2I)

Nägel (Fall 2, 9, I 4, I6)

Bleistifte (Fall 8, 10)

ro mal, $4 \mathrm{mal}$, 2 mal,

\begin{tabular}{|c|c|}
\hline Sonstige & $\begin{array}{l}\text { Io cm langes Stück von einem Münzen- } \\
\text { fänger (Fall } \mathrm{I} \text { ) }\end{array}$ \\
\hline längliche & Weidenzweig (Fall I 2 ) \\
\hline Gegen- & Löffel (Fall 3 ) \\
\hline & Fischgräte (Fall I I) \\
\hline
\end{tabular}

4 mal.

Diesen 20 Beobachtungen von Gegenständen mit überwiegenden Längsdurchmessern steht eine einzige Beobachtung (5) gegenüber, wobei es sich um ein verschlucktes Gebiß handelte ( $\mathrm{Ch}$ put). Zwei weitere von Wieting und Neugebauer mitgeteilte Beobachtungen von Ansammlung von Fruchtkernen im Duodenum nehmen insoweit eine Sunderstellung ein, als es sich um die Folge einer bestehenden Darmstenose handelte. Dieselben finden sich daher am Schlusse gesondert mitgeteilt.

Die genauere Lokalisation der Fremdkörper des Duodenum ist für I3 Fälle ersichtlich: 9 mal (Fall 2, 4, 6, 7, I I, I3, 14, I7, 21) gehört derselbe ausschließlich oder vorwiegend der Pars descendens an; je einmal ausgesprochen der Pars superior (Fall I5) bzw. dem Übergange der Pars inferior in den vertikalen Duodenalschenkel (Fall 8). In 2 Fällen wird schließlich als Sitz der Fremdkörper die Pars horizontalis inferior bezeichnet (Fall 5, 16).

Das Haupthindernis für die Passage langgestreckter Fremdkörper durch das Duodenum stellt also offenbarder Angulus inferior dar, d. h. der recht. 
winklige oder sogar spitzwinklige Übergang zwischen der vertikalen und der unteren horizontalen Duodenalportion. Der Flexuraduodeno-jejunalis, auf die Chaput früher einmal in diesem Sinne hingewiesen hat, kommt dagegen nur ausnahmsweise diese Bedeutung zu.

Eine praktisch schwerwiegende Folge, die sich unmittelbar aus dem oben skizzierten anatomischen Verhalten des Duodenums ergibt, ist die, daß außerordentlich leicht und frühzeitig dic Darmwand von einem derartig länglichen - häufig sogar spitzen - Fremdkörper perforiert oder sonst geschädigt wird.

So war unter 15 näher mitgeteilten Fällen eine komplet te Perforation des Darmlumens in 7 Fällen erfolgt (Fall I, 4, $6,7,12,13,16)$. Um eine beginnende Perforation handelte es sich in Fall 9 und 17 . Eine bloße Einkeilung bzw. EinspieBung in die Wand wurde in Fall 5, I I und I 5 verzeichnet. Daß auch in unserem Falle offenbar ähnliches mitgespielt hatte, lehren die bei der Operation gefundenen narbigen Veränderungen der Vorderwand der Pars superior. Ebenso ist auch die im Protokoll vermerkte chronische Pankreatit is wohl kaum anders zu erklären, als daß hier vorübergehend eine der Nadeln sich eingespießt und zur entzündlichen Reaktion in der Umgebung geführt hatte.

Am häufigsten scheint eine derartige Perforation im Bereichedes unteren horizontalen Duodenalschenkels vorzukommen. Es wird dies ohne weiteres verständlich, wenn man sich vergegenwärtigt, was geschehen muß, wenn ein langer Fremdkörper, der in der Pars descendens retiniert wurde, schließlich unter dem Einflusse der Peristaltik weiter nach abwärts gedrängt wird. Es kann dann eben leicht der Fall eintreten, daß das verschluckte Objekt nicht um die von dem Angulus inferior gebildete Kurve herumkommt, und die Folge wird sein, daß sich das vordere Ende desselben in die untere bzw. vordere Wand der Pars horizontalis einbohrt bzw. hier perforiert. Die Fälle 4, 6, 16 sind als Typus für diesen Hergang anzusehen. Ist die Länge des Fremdkörpers eine sehr beträchtliche und damit ein Übergang in den unteren Querschenkel des Zwölffingerdarms überhaupt ausgeschlossen, so kann 
gelegentlich auch die Perforation mit dem hinteren in der Pars superior gelegenen Ende erfolgen. Der von Buchstab mitgeteilte Fall (12) mag als Paradigma für diesen Modus gelten.

In diesem letzteren Falle drang der perforierende Gegenstand in die anliegende Leber ein; auch im Fall Curtillet (Nr. I3) wurde dies beobachtet. Im I all 16 fand sich schließlich bei der Sektion eine Nadel in der Lebersubstanz, die ebenfalls durch Perforation des Duodenums hierher gelangt war.

Wie schnell ein im Duodenum eingekeilter Fremdkörper eine schwere Schädigung der Darmwand herbeiführen kann, lehrt der von Geiling mitgeteilte Fall ( 17 ), wo die am nächsten Tage nach Eindringen des Corpus alienum in die Verdauungswege vorgenommene Laparotomie bereits die Zeichen der unmittelbar bevorstehenden Perforation erkennen ließ.

In anderen Fällen können Gegenstände sich jahrelang unbemerkt im Duodenum aufhalten, um erst viel später zu einer tödlichen Perforation zu führen.

Es gehört hierher folgender I892 von Libotsky mitgeteilter Fall:

Ein Weib im 8. Schwangerschaftsmonat erkrankt plötzlich unter den Erscheinungen einer akuten Peritonitis und stirbt. Bei der Autopsie findet sich eine dem $\mathrm{D}$ u o d e $\mathrm{n} u \mathrm{~m}$ angehörende Perforationsstelle; ein Löffel-den die Frau zwei Jahre vorher in selbstmörderischer Absicht verschluckt hatte - liegt frei in der Bauchhöhle (nach Perry und Shaw).

Zwei ähnliche Fälle von Duodenalperforation durch verschluckte Löffel sind von Froriep und Perry und Shaw mitgeteilt worden:

F roriep. Bei einem Wahnsinnigen, der unter den Zeichen der Peritonitis gestorben war, fand sich eine Perforation des Duodenums infolge eines im Zwölffingerdarm liegenden großen rostigen eisernen Löffels, den der Kranke, ohne daB man es wußte, verschluckt hatte (zit. bei $\mathrm{M}$ a yer, l. c.).

Perry und Shaw, I894.

Ein Geisteskranker verschluckte einen I I Zoll langen Löffel, mit dem Griffe voran. Derselbe passierte den Ösophagus und Magen, wurde aber an dem untersten $\mathrm{D}$ u ode $\mathrm{n}$ a $\mathrm{l}$ win $\mathrm{k}$ el festgehalten und führte dort zur Perforation mit nachfolgender Peritonitis. Das andere Ende des Löffels lag innerhalb des Pylorus.

Eine ähnliche Beobachtung findet sich schließlich bei $\mathrm{NuB}$ b a u m erwähnt: 
„Ein Gaukler verschluckte ein 9 Zoll langes Messer mit knöchernem Griff, hatte geringe Beschwerden. Nach drei Monaten starb er. Man fand den Griff im Magen aufgelöst und die Klinge sehr verkleinert, die Spitze im gangränösen Duodenum."

Ein Fall von Perforation des Duodenum durch einen verschluckten $\mathrm{Nagel}$ ist von Soyka mitgeteilt worden.

Eine Sonderstellung gegenüber den bisher erwähnten Fällen nimmt eine von Hahn mitgeteilte Beobachtung ein, wo die Perforation nach hinten zu erfolgte und zur Arrosion der Aorta mit tödlicher Blutung führte:

$\mathrm{H}$ a hn I9or. 28jährige Frau, vor 14 Tagen mit Schmerzen in der Magengegend erkrankt, seit drei Tagen Hämatemesis. Tod an zunehmender Anämie. - Sektion: $22 \mathrm{~cm}$ unterhalb des Pylorus ein fetziges Geschwür. In diesem sitzt ein borstenartiger, $3,5 \mathrm{~cm}$ langer $\mathrm{H}$ olzspan mit spitzen Enden, so daß nur $1 / 2 \mathrm{~cm}$ von demselben in das D u od en um hineinragt. Die Umgebung des Ulcus ist infiltriert, bis auf die Aorta, die eine linsengroße Perforation aufweist.

Daß aber auch die nicht penetrierenden Fremdkörperverletzungen der Duodenalwand gelegentlich äußerst folgenschwere sein können, lehrt der am Schlusse dieser Arbeit wiedergegebene Fall (I I) von Taylor und Lakin, wobei eine in die Duodenalwand eingespießte Fischgräte zu einer akuten, fortschreitenden, eitrigen Entzündung der Duodenalwand, einer sog. Duodenitis phlegmonosa, führte. Im einzelnen können wir hier auf diese seltene aber typische Erkrankung, über die erst kürzlich Frising und Sjövall eine ausführliche Darstellung gebracht haben, nicht näher eingehen.

$$
\text { Symptomatologie. }
$$

$\mathrm{Da} B$ ein Teil der im Zwölffingerdarm arretierten Fremdkörper, wenigstens zeitweise, völlig symptomlos verbleiben kann, ist sicher. Die im Stadium der Latenz befindlichen Fälle pflegen aber nur ausnahmsweise ärztliche Behandlung aufzusuchen, so daß man in der Regel bei den praktisch vorkommenden Fällen mit gewissen klinischen Erscheinungen zu rechnen hat.

Das wichtigste dieser Zeichen scheint in einer umschriebenen fixen, von der Lage der Fremdkörper abhängigen Druckschmerzhaftigkeit zu bestehen. Dem vor- 
zugsweisen Sitze derselben in der Pars descendens entsprechend, findet man diese druckempfindliche Stelle gewöhnlich - soweit wenigstens aus den spärlichen, in der Literatur niedergelegten Angaben hervorgeht -.. etwas rechts vom Nabel .... wic z. B. in unserem Falle - bzw. et was höher oben lokalisiert. In mehreren Fällen (7, I5) (English, Rovsing) wird ferner hervorgehoben, daß gleichzeitig Schmerzen bestanden, die nach dem Rücken bzw. der Schulter zu ausstrahlten. Auch in unserem Falle war dieses Phänomen ausgesprochen vorhanden und durch Druck von vorn her jedesmal hervorzurufen. Diese Rückenschmerzen waren es überhaupt gewesen, die unsere Pat. in ärztliche Behandlung geführt hatten. Im Falle Curtillet (I3) waren die Schmerzen sogar ausschließlich auf die Lumbalgegend lokalisiert. Es dürfte daher diesem Symptom eine gewisse Bedeutung für die topische Diagnostik zukommen. So spielt ja auch bei den traumatischen Duodenalrupturen das Phänomen der Schulter- oder Rückenschmerzen - vgl. Schumacher, 1. c. S. 494f. - eine gewisse Rolle, wie Geiling mit Recht hervorgehoben hat. Auch beim Ulcus pepticum duodeni kommen gelegentlich nach dem Rücken zu ausstrahlende Schmerzen vor.

Daß diese Rückenschmerzen von der Körperhaltung abhängig sein können, geht aus der Angabe unserer Pat. hervor, daß sie im Bett nicht mehr auf der Seite liegen konnte. Im Falle I3 (Curtillet) traten diese Schmerzen besonders bei dem Versuche der Rumpfbeugung nach vorne in die Erscheinung.

Sind die Schmerzen besonders hochgradige, so deutet dies wohlstets auf eine schwerere Läsion der Duodenalwand bzw. den Eintritt der Perforation hin. Es kommt dann schnell - wenn nicht noch in letzter Stunde rechtzeitig eingegriffen wird, wie in den Fällen 6 und 7 - zur allgemeinen Peritonitis, seltener zunächst zu umschriebener AbszeBbildung (Fall I6).

Klinisch imponieren diese Komplikationen seitens des Bauchfelles öfters zunächst als akute A ppendicitis (Fall 4, I I, I6). Es erklärt sich dies einmal durch die relative räumliche Nähe zwischen dem unteren Duodenalwinkel und der Ileocöcalgegend. Dann ist aber - namentlich auf Grund der Geschwürsperfora- 
tionen des Duodenum - längst bekannt ${ }^{1}$ ), daß die peritoneale Entzündung bzw. Eiterung in solchen Fällen sich in typischer Weise zu senken pflegt, und zwar entlang der Innenseite des Colon ascendens nach der Blinddarmgegend. Der Fall 16 von Hochhaus und Löschke illustriert dies in vollkommener Weise. Kommt ein derartiger Fall also erst in diesem sekundären Stadium zur Beobachtung und fehlen - wie so oft entsprechende anamnestische Angaben, so können gelegentliche diagnostische Irrtümer unvermeidlich sein. Es ist daher wohl denkbar, daß vielleicht manche ältere Fälle der Literatur, welche Fremdkörper betreffen, die aus Ileocöcalabszessen spontan oder operativ eliminiert wurden, nicht eigentlich in die Rubrik der Corpora aliena des Cöcums bzw. des untersten Ileums gehören, sondern vielmehr des Zwölffingerdarms. -

Obstruktionserscheinungen des Duodenums durch Fremdkörper der uns hier beschäftigenden Art werden nur ganz ausnahmsweise beobachtet. Als einziges sicheres Beispiel hierfür figuriert in der Literatur die nachstehende ältere Beobachtung von Marshall.

Marshall 1852. Pat. 8 Jahre nach dem Verschlucken einer größeren Quantität von Nadeln an Magenblutungen und Darmobstruktion zugrunde gegangen. Magen bis zur Symphyse dilatiert, enthält 9 Unzen Stecknadeln, dieselben sind nicht korrodiert, alle aber verbogen oder zerbrochen, viele sehr spitz. Der Magen fühlt sich an wie der Kropf eines Vogels. In dem wie mit Blei ausgestopften wurstartigen D u od en u m I Pfd. Nadeln (nach Wölf ler und Lieblein).

Ganz unsicher ist demgegenüber eine von $W$ eiBbart mitgeteilte Beobachtung anzusehen:

44jährige Frau, früher bereits Gallensteinkoliken durchgemacht, erkrankt mit heftigen Schmerzen rechts über dem Nabel. Erbrechen; Stuhl acholisch. Gänseeigroßer Tumor in der Gallenblasengegend palpabel. Nachdem dieser Zustand mit Intermissionen eine Reihe von Tagen angehalten hatte, verschwanden die Schmerzen schließlich nach einer besonders heftigen Kolik. Im Stuhl fand sich ein schmales Knochenstück von $3,5 \mathrm{~cm}$ Länge und durchschnittlich $\mathrm{I} \mathrm{cm}$ Breite.

I) Vgl. hierzu Melchior. Ergebnisse der Chirurgie 19II, Bd, 2, S. $242 \mathrm{ff}$. 
Wei $B$ bart deutet den Zusammenhang so, daß das Knochenstück eine partielle Obstruktion des Duodenums (Erbrechen) herbeiführte und anderseits durch Verlegung der Papille die Acholie der Fäzes sowie die Schwellung der Gallenblase bedingt habe. Sich einen derartigen Hergang praktisch vorzustellen, fällt indessen schwer. Eher wird man wohl annehmen müssen, $\mathrm{da} B$ es sich der Anamnese entsprechend um Cholelithiasis handelte, der verschluckte Knochen also nur eine zufällige Koinzidenz bedeutete. -

Über die Fremdkörperobstruktion des Duodenums bei gleichzeitiger organischer Stenose vergleiche weiter unten. -

Auf die Möglichkeit von Arrosionsblutungen wurde bereits oben hingewiesen.

\section{Radiologie.}

Als der wichtigste Faktor für die Erkennung von Fremdkörpern im Duodenum bzw. für ihre Lokalisation ist zweifellos das Röntgenverfahren zu bezeichnen. Es mögen hierzu einige Bemerkungen über das normale topographische Verhalten des Duodenums vorausgeschickt werden.

Nach Cornings Angaben (vgl. Fig. 2) liegt der Pylorus bei leerem Magen vor dem Körper des I. Lendenwirbels; die Pars superior schließt sich hieran quergestellt nach rechts. Die Pars descendens liegt rechterseits neben der Wirbelsäule in Höhe des 2. Lumbalwirbels; der Übergang in die Pars inferior erfolgt in Höhe des 3. Lumbalwirbels, der seinerseits von diesem Darmabschnitt überkreuzt wird. Das Endstück des Duodenums (Pars ascendens) steigt häufig wieder etwas proximalwärts auf, so daß die Flexura duodeno-jejunalis am linken Umfange des 2. Lendenwirbels angetroffen wird.

Die radiologischen Feststellungen ${ }^{1}$ ergeben durchschnitlich eine etwas tiefere Lage des Duodenums. So findet sich nach Saulé, dem wir hier folgen, der Pylorus normalerweise in der Mitte oder vor der linken Hälfte

I) Auf die Technik der Darstellung des Duodenums im Röntgenbilde gehen wir hier nicht ein. 
des 3. Lendenwirbels. Von hier steigt das Duodenum schräg nach oben rechts hinauf, bis zur Höhe des I. Lendenwirbels, die Pars descendens verläuft am rechten Rande der Wirbelsäule

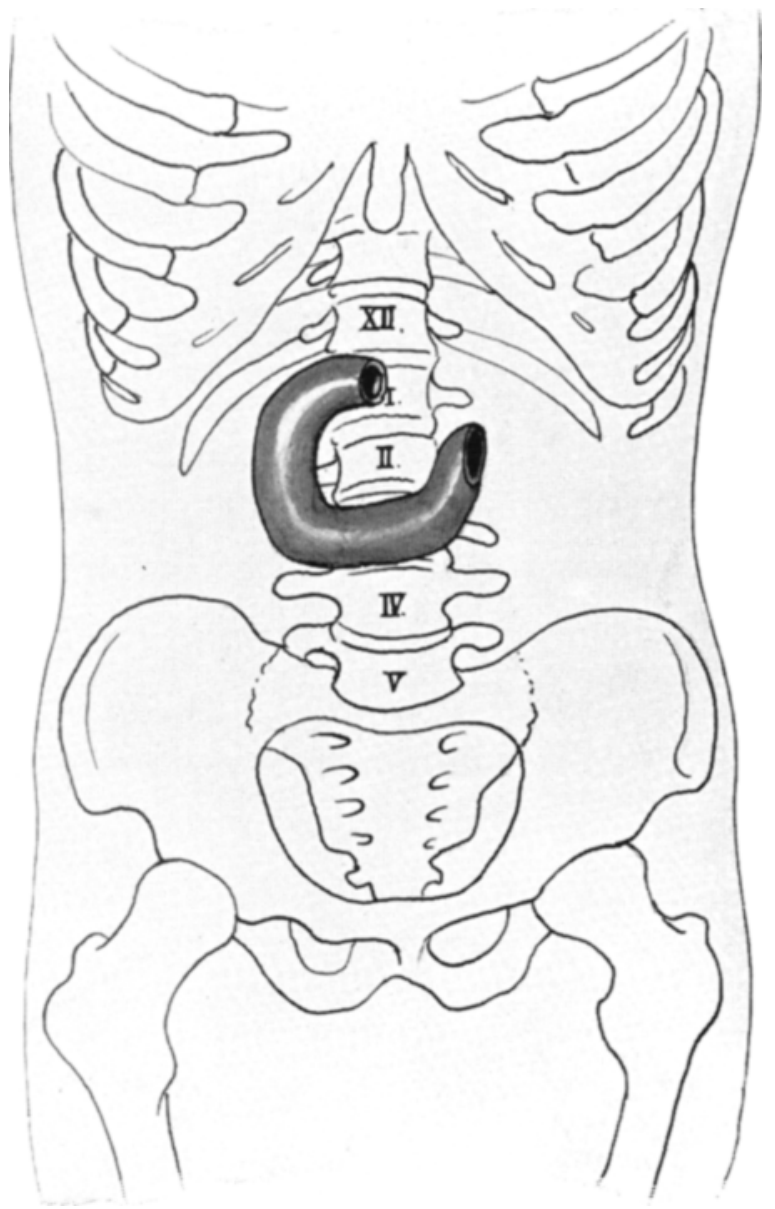

Fig. 2. Normale Situation des Duodenums (nach Angaben und in Anlehnung an eine Abbildung von Corning).

entlang bis zum Übergange in die Pars inferior, die horizontal vor dem Körper des 4. Lendenwirbels gelegen ist, um dann hier auch Pars ascendens genannt - schräg nach oben links aufsteigend in Höhe des 2. Lendenwirbels in die Flexura duo- 
deno-jejunalis überzugehen. - Allerdings ist hierbei zu berücksichtigen, daß diese radiologischen Feststellungen sich in cler Regel auf Untersuchungen am stehenden Menschen beziehen, wobei das Duodenum mit Ausnahme der fast absolut fixierten

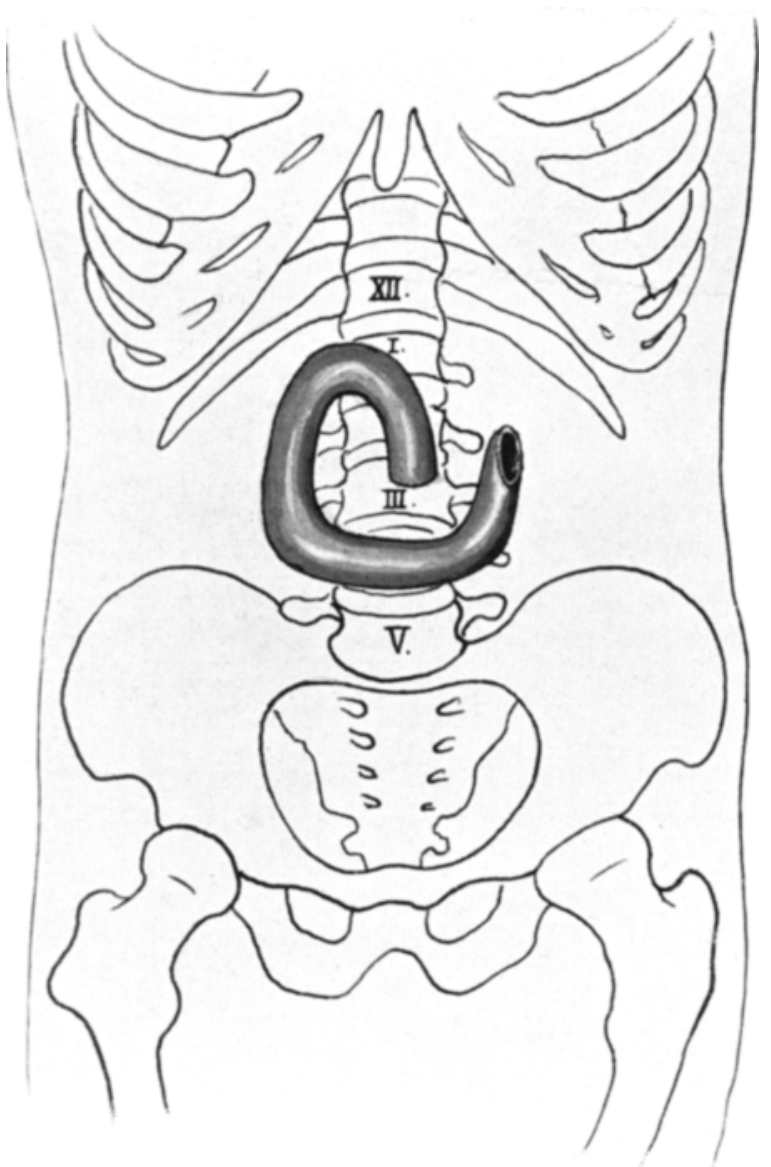

Fig. 3. Situation des Duodenums im Röntgenbilde (schematische Skizze nach Angaben von Saulél.

Flexura duodeno-jejunalis etwa um einen Wirbel tiefer tritt (Saulé). Andererseits sind aber gewisse Grade von Ptose des Duodenum überaus häufig. Auch Corning weist auf das nicht seltene Vorkommen eines Tiefstandes des Duodenum hin. 
Von eigentlichen Lageanomalien, die übrigens beim Duodenum nicht allzu selten sind, sei dabei abgesehen.

Wenn wir nun von der Erfahrungstatsache ausgehen, daß längliche Gegenstände - die hier ja fast ausschließlich in Betracht kommen - sich in der Regel in die Achsenrichtung des Duodenums einstellen, so folgt daraus, $d a ß$ verschluckte Fremdkörper, die an der rechten AuBenseite des 2. oder 3. bzw. auch noch des r. und 4. Lendenwirbels parallel zur Wirbelsäule im Röntgenbilde gelegen sind, mit größter Wahrscheinlichkeit auf das Duodenum, und zwar die Pars descendens zu beziehen sind. Das Colon ascendens liegt ja weiter nach außen von der Wirbelsäule, während Dünndarmschlingen rechts in dieser Höhe kaum in Betracht kommen, zumal wenn man die Aufnahme in Bauchlage anfertigt, wobei die nicht fixierten Darmpartien herabgedrängt werden. Eventuell würde sich bei schräger Durchleuchtung auch ein Anhalt für die Tiefe gewinnen lassen.

Tatsächlich haben nun die Röntgenaufnahmen in den bisherigen Fällen durchaus übereinstimmende Befunde ergeben. So fand sich im Falle Iñigo die Nadel rechts neben der Wirbelsäule; im Falle Schloffer war der in der Pars descendens befindliche Nagel rechts von der Mittellinie zu sehen; im Falle Geiling befand sich der Münzenfänger rechts von der Wirbelsäule vom I2. Brustwirbel bis 4. Lendenwirbel. In unserem Falle lagen die Nadeln annähernd senkrecht rechts vom 2. und 3 . Lendenwirbel. Eine größere Sicherheit kann man ev. noch gewinnen, wenn man den Magen mit Wismut füllt, um auf diese Weise seine topographischen Beziehungen zu den sichtbaren Fremdkörpern näher zu präzisieren (vgl. Fig. 4 unseres Falles).

Die Diagnose eines in der Pars inferior gelegenen Fremdkörpers wird in der Regel zu stellen sein, wenn das Röntgenbild denselben horizontal vor dem 4. bis 3. Lendenwirbel zeigt, und zwar namentlich, wenn er, wie in unserem Falle vgl. Fig. 5 - deutlich nach rechts herüberreicht ${ }^{1}$ ). Die ev, in

I) $\mathrm{Ob}$ die in diesem Bilde sichtbare Nadel im Verlaufe des zwischen der I. und 2. Röntgenaufnahme liegenden Monats spontan auf natürlichem 
Frage kommende Unterscheidung gegenüber einer Lokalisation im Colon transversum - die rein klinisch übrigens nicht sehr in Betracht kommt - würde durch Kontrastfüllung des Colons mittels Luftaufblähung wohl ohne weiteres durchzuführen sein. Dasselbe gilt bei einem mehr nach links gelegenen Fremdkörper gegenüber der ev. Unterscheidung von einem im Magen gelegenen Gegenstande. Im Falle Chaput - Gebiß im unteren Duodenum dicht vor der Flexura duodeno-jejunalis - war das-

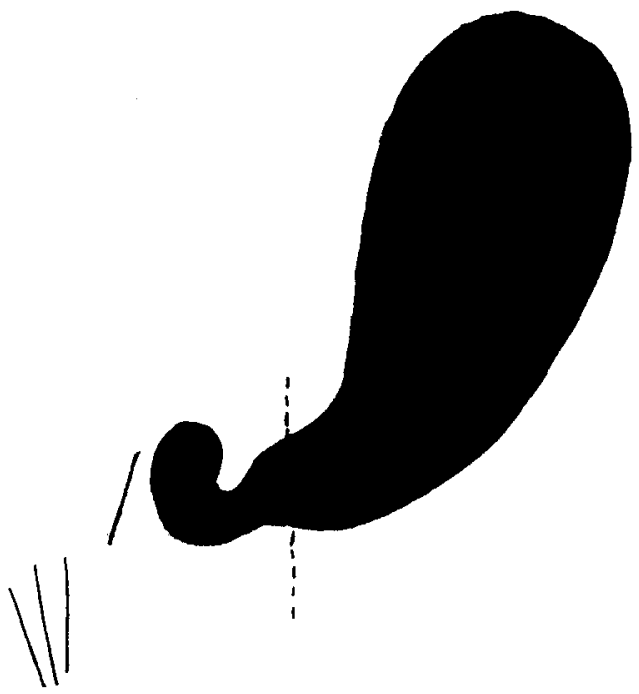

Fig. 4. (Verkleinerte Röntgenpause.)

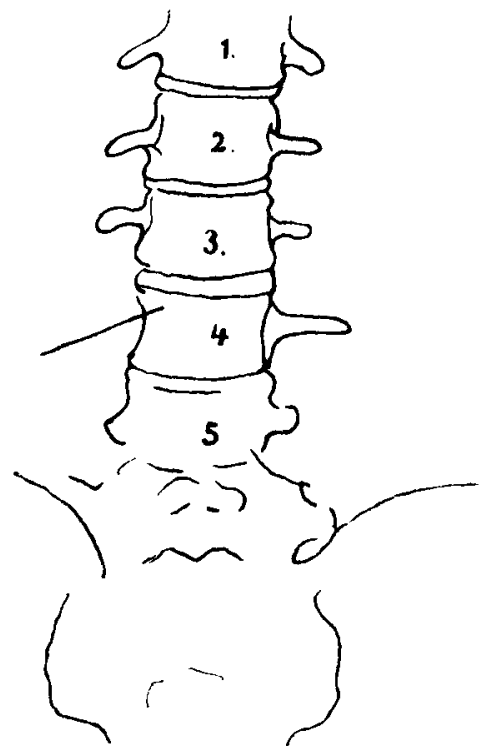

Fig. 5. (Verklein. Röntgenpause.)

selbe im Röntgenbilde an der linken Seite des 2. Lendenwirbels $\mathrm{zu}$ sehen.

Schwieriger kann die Deutung des Röntgenbefundes werden, wenn der betreffende Gegenstand bereits das Duodenum per-

Wege abgegangen ist oder vielleicht in die Pars descendens zurückgetreten ist, läßt sich nicht sagen, da die in der Pars descendens befindlichen Nadeln bei der ersten Aufnahme nicht sichtbar sind. Hieraus auf ein ursprüngliches Freisein der Pars descendens zu schließen, ist indessen nicht statthaft, da die Aufnahmen in Rückenlage offenbar für die Darstellung feinerer Fremdkörper des Duodenums wegen der größeren Entfernung von der aufnehmenden Platte weniger geeignet sind. 
foriert hat (vgl. Fall 6 und 7). Falls man jedoch in einem solchen Falle ursprünglich den Gegenstand in der Pars des. cendens lokalisieren konnte und derselbe späterhin weiter nach links schräg vor der Wirbelsäule nach abwärts verschoben erscheint, würde man allerdings schon rein nach dem Röntgenbilde eine Perforation annehmen können, soweit nicht schon der übrige Zustand direkt darauf hinweisen sollte.

Therapie.

Wir haben die diagnostische Seite dieser Frage etwas eingehender dargestellt, weil es nach den bisherigen Erfahrungen nicht ganz gleichgültig zu sein scheint, ob ein verschluckter Fremdkörper sich im Duodenum oder etwa im Colon ascendens oder transversum befindet.

Während man nämlich in letzterem Falle bei sonstigem Fehlen alarmierender Symptome sich in der Regel abwartend verhalten wird, erscheint bei den Fremdkörpern des Duodenums der bisher besprochenen Kategorie ein aktiveres Vorgehen angezeigt, angesichts der großen Gefahr einer ev. schon frühzeitig eintretenden Darmperforation. Läßt also das Röntgenbild als Sitz der verschluckten Nadeln, Nägel, Gebisse oder dergleichen das Duodenum erkennen, und zeigt sich bei mehrtägiger Beobachtung, daß das Corpus delicti keine Neigung hat, spontan weiter vorzurücken, so dürfte damit die Indikation zur operativen Entfernung gegeben sein. Bei stärkeren Schmerzen oder gar vorhandenen Zeichen einer entzündlichen Beteiligung des Peritoneums ist natürlich die sofortige Operation angezeigt. Ebenso wird es kaum zweckmäßig sein, länger zuzuwarten, wenn es sich um ein verschlucktes Objekt von solchen Dimensionen handelt, $\mathrm{da} ß$ ein ungestörter natürlicher Abgang a priori wenig wahrscheinlich ist. Daß natürlich der operative Eingriff wesentlich einfacher ist, wenn vorher eine präzise topische Diagnose gestellt werden konnte, liegt auf der Hand. So würde man sich z. B. in unserem Falle wohl kaum zu einer Mobilisierung des Duodenums - wodurch die Nadeln erst dem palpatorischen Nachweise zugänglich wurden - entschlossen haben, wenn man nicht schon von vornherein seiner Sache sicher gewesen wäre. 
Die Art der Operation wird sich nach dem Sitze des Fremdkörpers richten. Liegt derselbe hoch - also in der Pars superior bzw. dem Anfangsteil der Pars descendens -- so gelingt es gewöhnlich ohne weiteres - ev. nach Anhebung der Leber - denselben von außen her zu fassen und entweder durch direkte Incision oder besser vielleicht von einer Gastrotomie aus zu extrahieren. Falls die einfache Durchstechung möglich ist - wie in unserem von Fritsch operierten Falle, sowie dem von Curtillet -, so bildet dies natürlich das schonendste Verfahren. Um zu den tieferen Abschnitten der Pars descendens zu gelangen, kann die ebenfalls in unserem Falle geübte Mobilisierung des Duodenums von außen her sonst in der Regel nach partieller Durchtrennung des Lig. gastrocolicum - notwendig werden. In anderen Fällen kann es bequemer sein, nach Hinaufschlagen des Colon transversum und unter Durchtrennung des hinteren parietalen Peritoneums sich den Angulus inferior duodeni freizulegen.

Für Fremdkörper in dem untersten Duodenalabschnitt würde dieses Vorgehen sogar die Regel darstellen. Bezüglich aller technischen Einzelheiten dieser Zugänge sei auf die eingehende Darstellung bei Schumacher (1. c. S. 507 ff.) verwiesen. -

Die praktischen Resultate der wegen Fremdkörper vorgenommenen Operationen sind für 17 Fälle -von den am Schlusse gesondert mitgeteilten Beobachtungen von Wieting und Ne ugebauer abgesehen -- bekannt. Von diesen I 7 Fällen gelangten I I zur operativen Heilung, 6 starben. Wie weit ein Spättodesfall - Fall Iñigo - der Operation bzw. dem Grundleiden zur Last zu legen ist, ist mangels einer Sektion nicht zu sagen. Die relativ hohe Gesamtmortalität ist dadurch zu erklären, daB die meisten Fälle bereits als komplizierte zur Operation gelangten:

Eine vollständige Perforation der Darmwand lag in 6 Fällen vor (I, 4, 6, 7, I2, I3, I6), hiervon gelangten nur 2 zur Heilung. Ebenso ging der mit Duodenitis phlegmonosa komplizierte Fall I I zugrunde. Es endeten ferner von 2 als beginnende Perforation (9, I7) aufzufassenden Fällen einer (17) tödlich. Die unkomplizierten Fälle gelangten dagegen sämtlich zur operativen Heilung. 
Die im voranstehenden vertretene Forderung der frühzeitigen Operation bei Fremdkörpern des Duodenums erhält durch diese Erfahrungen eine wesentliche Stütze.

Kasuistik.

Wir schließen hieran die Wiedergabe der einzelnen im Texte näher berücksichtigten Beobachtungen:

Fall I. Kränzle (v. Bruns) Igor.

24jähriges Mädchen - Melancholie - verschluckt seit 21/2 Jahren Zaunnägel, Stricknadeln, Knopfhaken usw. Häufig wiederkehrende Anfälle von Schmerzen und Erbrechen, in den letzten Wochen fast andauernd. Einzelne verschluckte Gegenstände sind im Laufe der Zeit spontan abgegangen. Patientin ist sehr heruntergekommen, in der Pylorusgegend eine undeutliche Resistenz palpabel. Epigastrium ist stark druckempfindlich. Zahlreiche Fremdkörper im Röntgenbilde. Ein Konvolut derselben in der rechten Körperhälfte zwischen Darmbeinkamm und II. Rippe. La pa ro tomie. Mittels Gastrotomie werden eine Anzahl Fremdkörper aus dem Magen extrahiert, schwieriger gelingt es unter vorsichtiger Mobilisation, zwei im $\mathrm{D}$ u odenum in die vordere und hintere Wand eingespießte, quer zur Längsachse des Darms stehende Gegenstände - eine Nadel sowie einen Knopfhaken - zu entfernen. Insgesamt werden 25 Fremdkörper entfernt. Exitus nach 5 Tagen an eitriger Peritonitis, ausgehend vielleicht von einer unter der Einwirkung der eingespießten Fremdkörper stattgefundenen - allerdings durch die Leber gedeckten - Perforation des Duodenums. Die ganze Duodenalschleimhaut war durch Schwefeleisen schwarz verfärbt.

Fall 2. Lucas 1903.

22 Monate altes Kind verschluckt einen 2 Zoll langen Nagel. Das Röntgenbild zeigt denselben rechts von der Wirbelsäule in Höhe des I. bis 4. Lendenwirbels. Nachdem derselbe I Monat lang in derselben Lage verharrt hat, wird die $L$ a p a r o t o m i e vorgenommen und der in der Pars descendens duodeni befindliche Nagel durch Incision des Darms entfernt. Heilung.

Fall 3. Revenstorf (L a uenstein) 1906.

Ein 26jähriger, anscheinend geisteskranker Mann hatte einen $L$ öf f el verschluckt. Im Röntgenbild ist derselbe deutlich sichtbar, der Stiel liegt schräg in der rechten Oberbauchgegend und überragt die Wirbelsäule nach links um einige Millimeter. Gastrotomi e (L a u enstein). Im Magen findet sich neben dem $\mathrm{I} 3,5 \mathrm{~cm}$ langen Stiel eines Metallöffels ein Holzlöffelstiel von gleicher Länge. Beide ragen mit ihren Enden in das D u odenum hinein. Magennnaht, Heilung. Nachträglich ergibt sich, daß Patient bereits vor einem Jahr 
aus dem gleichen Anla $B$ operiert worden war; es fand sich damals je ein Löffelstiel im Magen und Duodenum, der letztere muBte durch eine Incision des $Z$ wölffingerdarms selbst entfernt werden.

Fall 4. Mennacher igó.

6jähriges Mädchen, vor 4 Monaten eine $\mathrm{H}$ a a rnadel verschluckt, anfangs beschwerdefrei, plötzlich mit Leibschmerzen und Erbrechen erkrankt, Bauch bretthart gespannt. Besonders druckempfindlich scheint die Blinddarmgegend zu sein; zunächst Verdacht auf Appendicitis. Das Röntgenbild zeigt die Haarnadel schräg vor der Wirbelsäule liegend, mit der Spitze rechts oben in der Höhe des unteren Randes des 2. Lendenwirbels, das stumpfe Ende befand sich unten vor der linken Hälfte des Promontoriums. Operation am 2. Tage (L uxenburger): Diffuse, fibrinös eitrige Peritonitis. ,die Haarnadel hatte mit dem stumpfen Ende das D u odenum am Übergang der Pars descendens in die Pars ascendens samt der dahinterliegenden Radix mesenterii durchstochen und ragte, ohne daß sich hier Verklebungen gebildet hatten, hinten vor der Wirbelsäule in die freie Bauchhöhle". Extraktion. Enteroraphie. Tod in der folgenden Nacht.

Fall 5. Chaput 1907.

Eine Frau verschluckt ein GebiB; Schmerzen in der Median. linie des Bauches; das R a di og ramm zeigt den Fremdkörper an der linken Seitc des 2. Lendenwirbels. L a pa r o t o mie am i I. Tage: Magen und Dünndarm leer; erst bei Hochschlagen des Colon transversum und Herabziehen des Dünndarms fühlt man das Gebiß im unteren Querstück des Duodenums gerade vor der Wirbelsäule fixiert. Extraktion schwierig, da der Fremdkörper sich in die Darmwand fest eingekeilt hat. Darmnaht. Heilung.

Fall 6. Dehmel igio.

5jähriger Knabe, vor 4 Tagen eine $\mathrm{N}$ a d el verschluckt. Druckschmerzhaftigkeit rechts oberhalb des Nabels.

Röntgenphotographie: $6 \mathrm{~cm}$ langer scharfer Schatten einer Nadel mit kirschkerngroßem Knopf. Die Nadel liegt bei ventrodorsaler Durchleuchtung so, daß der Schatten des Nadelknopfes in Höhe der rechten I2. Rippe sich befindet, indem er - von der Rippenspitze an gerechnet - am Ende des ersten Drittels des Rippenschattens liegt. Der Schatten verläuft dann in schräger Richtung zur Lendenwirbelsäule, die Nadelspitze erreicht die Radix des Arcus vertebrae lumbal. IV. Mediane L a p a r o t o m i e (H a e ckel). Keine Peritonitis. Nadelknopf im Duodenum palpabel, die Spitze ragt etwa $3 \mathrm{~mm}$ aus der Radix mesenterii heraus, in der Umgebung eitrige Belege. Incision, Extraktion, Darmnaht. Gazetamponade. Nach 3 Wochen geheilt entlassen. 
Fall 7. English igro.

Ein I6jähriges Mädchen verschluckt eine zerbrochene Schnürnadel (,bodkin"), als sie im Begriffe war, sich mit derselben die Zähne zu reinigen. Seitdem ein stechender Schmerz sowie eine geringe Druckempfindlichkeit im rechten Hypochondrium bei sonstigem Wohlbefinden. Das Röntgenbild zeigt den Fremdkörper rechts vom Nabel, offenbar dem Magen oder Duodenum angehörend. Es wurde zunächst abgewartet; als sich aber am 13. Tage plötzlich heftige Schmerzen, nach der rechten Schulter zu ausstrahlend, und Muskelspannung einstellten, ergab sich die Indikation zur sofortigen $O$ peration: Incision durch den rechten Rektus. Peritoneum injiziert; die Nadel ragt frei aus der Pars descendens $\mathbf{d}$ u o de $\mathrm{n}$ i heraus. Extraktion. Darmnaht. Gazetampon, Heilung. Die Länge der zerbrochenen Nadel betrug 21/2 Zoll; die Perforation war mit dem ab. gebrochenen Ende erfolgt.

Fall 8. Hastings I9Io.

3jähriges Mädchen verschluckt einen mit einer Metallhülse versehenen Bleistift; am nächsten Tage kolikartige Schmerzen; das Röntgenbild zeigt den Fremdkörper in einer Entfernung von I $1 / 2$ Zoll von der Mittellinie unterhalb des rechten Rippenbogens. Da bei wiederholten Aufnahmen der Fremdkörper unverrückt an Ort und Stelle bleibt, wird am 14. Tage die O per at io n vorgenommen. Mediane Laparotomie: Die eingeführte Hand fühlt den Bleistift quergestellt im $\mathrm{D}$ u o d e $\mathrm{n} \mathrm{u} \mathrm{m}$ am Übergang der Pars descendens in die Pars horizontalis inferior. Derselbe wird mobilisiert und in den Magen zurückgebracht. Gastrotomie, Extraktion, ungestörte Heilung. Der Bleistift war $17 / 8$ Zoll lang und betrug $3 / 16$ Zoll im Durchmesser.

Fall 9. Schloffer igII.

35jähriger Mann verschluckte bei der Arbeit eine größere Anzahl von $8 \mathrm{~cm}$ langen Nägeln, die er im Munde hielt. Seitdem bald da, bald dort stechende Schmerzen im Bauch, Allgemeinbefinden nicht nennenswert gestört. Die Röntgenuntersuchung ergibt das Vorhandensein mehrerer Nägel im Darmtraktus, von denen nach Kartoffeldiät einer spontan abgeht. Drei Wochen später stellen sich leichte Schmerzen in der B linddarmgegend ein, am folgenden Tage Temperatur von 39,2 Grad, Druckschmerzhaftigkeit des Bauches. Aufstoßen. Die Röntg enuntersu chung zeigt jetzt einen Nagel rechts, einen anderen links von der Mittellinie. Operation: Es findet sich zunächst ein $8 \mathrm{~cm}$ langer Nagel in einer frei beweglichen Dünndarmschlinge vor. Extraktion. „Beim weiteren Nachsuchen fand sich im unteren Teile der Pars descendens duodeni, deren vordere Wand mit fibrinösen Auflagerungen bedeckt und an einer Stelle verdickt war, bei der Palpation noch ein weiterer Nagel, der wiederum durch einen kleinen Einschnitt extrahiert wurde. Hierbei zeigte sich, 
daß an dieser Stelle zwei Nägel knapp nebeneinander lagen, wesentlich dünner als der zuerst entfernte Nagel." Enteroraphie. Heilung.

Fall ro. Pierce igri.

Bezüglich des Falles von Pierce liegt nur die kurze Notiz vor: „Bleistift aus dem Duodenum entfernt (Demonstration)."

Fall II. Taylor und Lakin I9II.

66jährige Frau, plötzlich vor 36 Stunden unter abdominellen Erscheinungen erkrankt, als Appendicitis eingeliefert. Auftreibung und Druckschmerzhaftigkeit rechts von der Mittellinie oberhalb der eigentlichen Appendixgegend.

Sofortige Operation: Appendix intakt. Pankreas und Duodenalschlinge ödematös geschwollen, mehrere Incisionen in das Drüsenparenchym ergeben keinen Eiter. Drain, Schluß der Bauchwunde. Unter anhaltendem Erbrechen Tod 36 Stunden post operationem.

Sektion: Peritonitis acuta purulenta. Die 2. Duodenalportion erscheint stark verdickt, die Serosa fibrinös beschlagen. Submucosa eitrig infiltriert mit Abhebung der Schleimhaut. Stellenweise bildet die Mucosa bis haselnußgroße Erhebungen. Inmitten der infiltrierten Zone befindet sich eine zum Teil in das Darmlumen frei hineinragende Fischgräte. Pankreaskopf geschwollen, keine Fettnekrose.

$\mathrm{B}$ akteriologis ch enthält der Eiter Bacterium coli in Reinkultur, derselbe Erreger findet sich in dem nebenher bestehenden fibrinösen pleuritischen Exsudate.

Fall i2. Buchstab I9I2.

I6jähriger Knabe verschluckte vor 5 Monaten einen $15 \mathrm{~cm}$ langen Weidenzweig. Nach 3 Wochen periodische Leibschmerzen, nach 2 Monaten Fieber, zuletzt Husten und Atemnot. Geschwulst unter der Leber - hinter dem Magen? Laparotomie: Hinter dem stumpf durchtrennten kleinen Netz stinkender Eiter. Fremdkörper nicht gefunden. Tamponade. Tod am selben Tage. Die S ekt i o n zeigte multiple Abszesse im Lobus Spigelii; der Zweig saß im D u od e n u m festgekeilt, das eine Ende im unteren horizontalen Schenkel, das andere hatte die obere Wand der Pars superior durchstochen und $\mathrm{saB}$ in einer festen Narbe in der Substanz des linken Leberlappens.

Fall i3. Curtillet igiz.

40jähriger Mann verschluckt eine $10 \mathrm{~cm}$ lange aufgebogene, sehr feine, an einem Ende mit einem Wattetupfer armierte $\mathrm{H}$ a a $\mathrm{r}$ a del. Zwei Tage später leichte Schmerzen in der L u m b a lg e g en d, etwas nach außen von der Wirbelsäule, die sich in der Folge verstärkten und besonders heftig wurden, wenn sich der Kranke nach vorn beugte. Röntgenbild negativ. La pa ro to mie am 6. Tage. Das Colon as- 
cendens, an das wegen des Sitzes der Schmerzen zunächst gedacht wird, ist frei, ebenso das Quercolon und der absteigende Dickdarm. Beim Anheben der Leber wird schließlich die Nadel sichtbar, welche die Duodenalwand durchspießt hat und $3 \mathrm{~cm}$ tief in die Leber hineinragt. Durch weiteren Zug gelingt es, den Fremdkörper mitsamt der Watte aus dem Darm zu extrahieren. Auf Nahtverschluß der feinen Öffnung wird verzichtet. Abstopfen. Drainage. Komplikationslose Heilung.

Fall I4. Iñigo I9I3.

„Ein $21 / 2$ Jahre altes Mädchen verschluckte eine $12 \mathrm{~cm}$ lange spitze Nadel, die gar keine Erscheinungen machte. Durch die wiederholte röntgenologische Untersuchung wurde festgestellt, daB die Nadel oberhalb des Nabels, senkrecht stehend, rechts neben der Wirbelsäule zu sehen war. Da nach 8 Tagen die Lage des Fremdkörpers sich nicht geändert hatte, wurde die Laparotomie ausgeführt und die Nadel aus dem absteigenden Abschnitt des Duodenums entfernt. Die dazu nötige Darmöffnung wurde mittels zweireihiger Naht geschlossen. Die Heilung erfolgte glatt, jedoch ging die Kranke sechs Wochen nach der Operation an einer plötzlich einsetzenden Peritonitis zugrunde. Die Sektion konnte nicht ausgeführt werden."

Fall 15. Rovsing 1913.

„ı Ijähriger Knabe. Periodische Schmerzen im Rücken während 3 Wochen nach dem Verschlucken einer Nadel (Sicherheitsnadel). Laparotomie. Die Nadel wurde im Duodenum, mit dem abgerundeten Ende gegen den Pylorus und der Spitze in der hinteren lateralen Wand des Duodenums gefunden. Heilung."

Fall I6. Hochhaus und Loeschke I9I3.

Der Fall betrifft einen Mann, der wegen Verdacht auf Blinddarmentzündung zur Einlieferung kam. Patient gab an, daß er kurze Zeit vorher mit Schmerzen im Leibe erkrankt sei. Bei der Untersuchung war der Leib weich, etwas oberhalb der Ileocöcalgegend war eine druckempfindliche Stelle; das Befinden war ein leidlich gutes, das Fieber gering. Zwei Tage nach der Aufnahme trat ein sehr heftiger Schüttelfrost ein mit nachfolgendem Fieber, deshalb wurde der Kranke der chirurgischen Abteilung überwiesen und gleich operiert. Es fand sich hierbei ein Abszeß rechts neben der Wirbelsäule, der sich hinauf bis zu dem Du ode $\mathrm{num}$ verfolgen ließ, das eine große Perforation zeigte. Nach der Operation trat anfangs Wohlbefinden ein; der Kranke erlag dann aber einer Pneumonie.

Die Sektion ergab eine diffuse Peritonitis; als Ausgangspunkt derselben eine Perforation des Duodenums dicht oberhalb der Durchtrittsstelle unter der Radix mesenterii. Diese Perforation war hervorgerufen durch einen etwa $6 \mathrm{~cm}$ langen $\mathrm{K}$ istennagel, der noch zum Teil innerhalb des Darms lag. Es hatte sich von hier aus zuerst eine abgekapselte Eitersenkung der Radix mesenterii ent- 
lang bis in die Ileocöcalgegenà gebiidet, die später zur diffusen Peritonitis geführt hatte.

Außer diesem Nagel fand sich im M a g e $n$ der Leiche cine Nähnadel und im Leberhilus eine zweite Nadel, die hier durch sehr derbes Bindegewebe fixiert war. Verwachsungen und Narben wiesen darauf hin, daß diese Nadel ihren Weg in die Leber durch die Wand des.Duodenums gefunden hatte.

Irgendwelche Anhaltspunkte für die eigenartige Ärtiologie dieses Befundes hatte dic Anamnese nicht ergeben.

Fall i7. Geiling I9I3.

$2^{1 / 2}$ jähriger Knabe verschluckt eine Münze. Beim Versuch der lixtraktion bricht der untere -- ca. Io $\mathrm{cm}$ lange -- Teil des Instruments ab. Extraktion der Münze mittels Ösophagoskops; der Münzenfänger scheint sich - wie die Durchleuchtung ergibt -- im Magen zu befinden. Am nächsten Tage zeigt sich derselbe $r e c h t s$ von der Wirbelsäule, etwa vom 12. Brustwirbel bis 4. L.cndenwirbel; das obere spitze Ende liegt näher der Wirbelsäule als das untere breite Ende. Da befürchtet wird, daß der Fremdkörper den Magen durchspießen könne, Operati on: Mediane Laparotomie, Magen frei. Das obere Ende befindet sich in der Pars hor. sup. duodeni, das untere in der Pars descendens. „Die Stelle, wo die Spitze in der Pars superior steckt, war auch von außen ... daran zu erkennen, daß hier cine weißliche Verfärbung der Serosa bestand. Die Darmwand fühlt sich verdünnt an." Zurückschieben des oberen Endes in den Magen, das untere folgt erst nach Dehnung des Pylorus. Gastrotomic. Extraktion. Gastroraphic. Cbernähung der geschädigten Stelle der Duodenalwand. Tod am nächsten Tage an Aspirationspreumonie.

\section{Fall i 8 und 19. La n $z$ (ohne Jahr).}

Wie Geiling angibt (l. c., S. 26):,.... ist es nach einer persönlichen Mitteilung von Prof. La $\mathrm{n} z$, Amsterdam, diesem in letzter Zeit gelungen, in zwei Fällen Sicherheitsnadeln aus dem Duodenum in den Magen zu befördern, und hier nach Magenschnitt zu extrahieren."

Fall 20. Bataryal 1913 .

Hutnadel im Duodenum. Keine näheren Angaben, siehe Literaturverzeichnis.

Fall 21. Persönliche Bcobachtung (Op. Frits $\mathrm{ch}$ ) siehe vorn.

Eine völlige Sonderstellung neben den übrigen Fällen nehmen einige weitcre, zum Teil bereits oben erwähnte Bcobachtungen ein, in denen die Arretierung von Fremdkörpern -.. in Gestalt von Obstkernen -- auf eine bereits bestehende organische Stenose des Duodenums zurückzuführen war. 
Fall 22. Wieting und Effendi.

25jähriger Mann, Erscheinungen einer hochsitzenden Darmstenose.

Operation: Magen stark gebläht, das Hindernis befindet sich im Duodenum, man fühlt daselbst eine große Menge harter knirschender Körper, die als Gallensteine angesprochen werden. Gastroenterostomic. Plötzlicher Tod am Schluß der Operation.

Sektion: Hochgradige suprapapilläre narbige Duodenalstenose, Duodenum oberhalb auf etwa $300 \mathrm{ccm}$ Inhalt dilatiert, der Magen faßt 5 Liter Flüssigkeit. Die gefühlten Fremdkörper stellen Olivenkerne - etwa $80-90$ an Zahl - dar; dic Passage war dadurch annähernd vollständig verlegt.

Ein ähnlicher Fall ist von Neugebauer (persönliche Mitteilung) beobachtet worden:

„Duodenalstenose, etwa in Gegend der Papilla. Das zuführende Duodenum war mächtig erweitert. In diesem Sack schepperten Steine, wie sich später herausstellte, verschluckte $P$ flaumen. k e r n e, welche das verengte Lumen nicht passieren konnten. Gastroenterostomie. Heilung."

In eine gleiche Kategorie gehört möglicherweise auch noch eine ältere, uns im Original nicht zugängliche Beobachtung von Jaymes (1780), die den Titel trägt: „Observation sur les effets funestes des noyaux de prune avalés et arrêtés dans le duodénum".

Es schließt sich hieran folgende von Schmorl leider nur ganz summarisch und ohne klinische Angaben mitgeteilte Beobachtung :

„Enorme Dilatation des Duodenums, kein Passagehindernis, der dilatierte Teil mit gewöhnlichem Quarzsand gefüllt; wie dieser in den Darm gekommen ist, war nicht mehr nachweisbar. Der ganze andere Darm war sandfrei, aller Sand hatte sich hinter einem Sporn der Duodenalschleimhaut gefangen."

Es sei schließlich noch erwähnt, daß kleinste Fremdkörper, wie feine Knochensplitter, Traubenkerne usw. gelegentlich in den nicht selten vorkommenden, gewöhnlich in der Umgebung der Papille sich findenden Divertikeln des Duodenums aufgehalten werden können. Ob dies immer als harmloses Ereignis aufzufassen ist, möchten wir dahingestellt sein lassen. Eine Durchsicht der in der Literatur niedergelegten Fälle scheint uns 
vielmehr darauf hinzuweisen, daß von derartigen retinierten Fremdkörpern aus gelegentlich eine eigentliche Diverticulitis bzw. selbst eine Phlegmone der Darmwand - entsprechend der bereits erwähnten Duodenitis phlegmonosa - ausgehen kann. Auf Einzelheiten müssen wir an dieser Stelle verzichten.

\section{Literaturverzeichnis.}

I. Batavya 1, K. L., A case of hat-pin in the duodenum. Ind. med. gaz. I9ı3, Bd. 48, p. I48. Zit. ohne nähere Angaben im Zentralbl. f. d. ges. Chir. 19I3, Bd. I, S. 757 .

2. Buchstab, J. A., Zur Kasuistik der Fremdkörper im Darm. Russki Wratsch I9I2, Nr. 38. Ref. Zentralbl. f. Chir. I912, Nr. 48, S. I661.

3. Chaput, Corps ćtranger de la terminaison du duodénum. Dentier ayant traversé l'oesophage et l'estomac et arrêté à la terminaison du duodénum; enlevé par entérotomie. Bull. et mém. de la soc. de chir. 1907, p. 1217 .

4. Corning. Lchrbuch der topographischen Anatomic Igog. 2. Aufl.

5. Curtillet, Corps étranger du duodénum (tige métallique). Soc. de Méd. d’Algcr. I912, Februar. Presse méd. I9I2, Nr. 62, p. 647.

6. Dehmel, Eine Nadel im Duodenum durch Enterotomie entfernt. Deutsche med. Wochenschr. I9I0, Nr. 44, S. 2049.

7. English, T. Crisp), Perforation of the duodenum by a bodkin; laparotomy, recovery. Lancet 1905 , II, p. I 545.

8. Frising, G., u. Sjövall, E., Die phlegmonöse Enteritis im Duodenum und im Anîangsteil des Jejunums. Jruns' Beitr. 1913, Bd. 83, S. I.

9. Ga ulti c r, René, Les maladies du duodénum. Paris (Baillière) Igro.

Io. Geiling, Eugen, Zur Kasuistik der Fremdkörper im Duodenum. Inaug.-Diss. Leipzig I913.

II. Hahn, Demonstration zweier Präparate von Ulcus duodeni. Freie Vereinigung der Chirurgen Berlins. I2. XI. I900. Zentralbl. f. Chir. I90I, S. 22.

12. Hastings Sommerville, Foreign bodies in the duodenum. Brit. med. Journ. igio, I, p. 370.

I3. Jaymes, Observation sur les effets funestes des noyaux de prune avalés et arrêtés dans le duodénum. Journal de méd. chir. et pharm. etc. Paris 1780 , p. 424.

I4. I ñ i o, Fremdkörper im Duodenum (spanisch). La clínica moderna. I2. 1913, p. 269. Ref. Zentralbl. f. d. ges. Chir. I9I3, II, S. 208.

I5. Kränzle, Die Entfernung von Fremdkörpern aus dem Magen durch Gastrotomie. Bruns' Beitr. I90I, 29, S. 327.

16. Libotsky. Medical Press 1892. 16. XI. Zit. nach Perry und Shaw. 17. Löschkc, Demonstration der Organe eines Fremdkörperschluckers. 
(Perforation des Duodenums durch einen Kistennagel.) Rheinisch-westfälische Gesellschaft für innere Medizin, Nervenheilkunde. 6. VII. I9I3. Münchn. med. Wochenschr. I913, S. 2378. (Klinischer Teil von H o ch h u s.)

18. L u cas, Clement, Reports of the society for study of diseases of children. I903, p. 272. Zit. nach H as $t$ ings.

19. Marshall. Dublin med. Press 1852. Zit. nach Wölfler und Lieblein.

20. M a y e r, A., Die Krankheiten des Zwölffingerdarms. Düsseldorf 1844 . (S. d. S. 52: Fall Frorie p.)

21. Mennacher, Perforation des Duodenums durch eine verschluckte Nadel. Münchn. med. Wochenschr. 1906, S. 2570.

22. $\mathrm{NuBbaum,} \mathrm{v.,} \mathrm{Die} \mathrm{Verletzungen} \mathrm{des} \mathrm{Unterleibes.} \mathrm{In:} \mathrm{Deutsche}$ Chirurgie I880, Liefg, 44. (S. d. S. 51 .)

23. Perry, E. C., u. Shaw, L. E., On diseases of the duodenum. Guy's Hospital Reports 1894, vol. L, p. 171.

24. P i e r c e, Bedford, Lead pencil removed from the duodenum. (Demonstration.) Leeds and West Riding med.-chir. Soc. 20. I. I9I I. Brit. med. Journ. I9II, I, p. 309.

25. Revenstorf, Gastrotomie behufs Entfernung von Fremdkörpern. Biologische Abteilung des ärztlichen Vercins Hamburg. I2. III. 1906. Münchn. med. Wochenschr. I906, S. 1232.

26. Rovsing, Th., Corpus alienum duodeni (Sicherheitsnadel). Dänische chirurgische Gesellschaft. 23. Sitzung vom I8. XI. I9II. Zentralblatt für Chirurgie 1913, Nr. 27, S. I07 I.

27. Saulé, Les ptoses du duodénum. Thèse de Lyon (Maloine éd.) I912.

28. S chloffer. Wissenschaftliche Ärztegesellschaft in Innsbruck. I8. V. I9II. Wiener klin. Wochenschr. 19II, S. I5I3.

29. Sch u m a cher, E. D., Zur Duodenum-Chirurgic. Bruns' Beitr. 1910, Bd. 7 I, S. 482 .

30. $\mathrm{Sch}$ m orl. Geselischaft für Natur- und Heilkunde zu Dresden. Sitzung vom I. IV. I9I I. Münchn. med. Wochenschr. I9ri, Nr. 24, S. 1326.

3 ז. Soyka, Perforation des Duodenums durch einen verschluckten Nagel. Prager med. Wochenschr. I 876, I, S. 509.

32. T a y lor, Gordon, u. L a ki n, C. Ernest, A fatal case of phegmonous inflammation of the duodenum following impaction of a fish bone. The Lancet I9II, II, p. 224 .

33. Weissbart, Zur Kasuistik der Fremdkörper in Magen und Darm. Münchn. med. Wochenschr. I906, S. 703.

34. Wieting u. Effendi, Zur Pathologie und Anatomie der suprapapillären Duodenalstenose auf geschwüriger Grundlage. Deutsche med. Wochenschr. 1904, Nr. 53, S. 1953.

35. Wölfler, A., u. Li e ble in, V., Die Fremdkürper des Magen-Darm. kanals des Menschen. Deutsche Chirurgie 46 b. Stutgart I909. 